\title{
Comparison between experiment and computer modelling of plane-strain simple-shear ice deformation
}

\author{
C.J. L. WILSON \\ School of Earth Sciences, University of Melbourne, Parkville, Victoria 3052, Australia \\ Y. ZHANG \\ CSIRO, Division of Exploration and Mining, Private Mail Bag, Wembley, Western Australia 6014, Australia
}

\begin{abstract}
An examination of both experiments and computer models of polycrystalline ice undergoing a simple shear suggests that there is good agreement. The model has correctly reproduced the deformational and microstructural features caused by glide on (0001) in the ice aggregates. This success is particularly prominent for those ice grains with a lattice orientation suitable for hard or easy glide or kinking, and where there is a sub-horizontal $c$ axis and a larger grain-size. A limitation may be that the model cannot explicitly simulate recrystallization and grain-boundary migration, which are two other important processes operating jointly with glide in experimentally deformed ice. However, through the use of the models, it is possible to show how kinematic factors can control the processes of recrystallization. The localization of recrystallization in the polycrystalline ice aggregate is determined by the stress and strain variations between neighbouring grains.
\end{abstract}

\section{INTRODUCTION}

Shear strain is the dominant contributor to the flow of most natural ice masses (Hooke and Hudleston, 1978; Lliboutry, 1987). Despite the large amount of work that has been done on the mechanical properties of polycrystalline ice, our understanding of this material on a grain scale has many serious gaps. For instance, it is well known that ice deforms dominantly on the basal plane (Weertman, 1983; Fukuda and others, 1987) but its relationship to the important processes of grain-boundary migration is subject to much speculation (e.g. Alley, 1992). In order to facilitate an understanding of the complex processes involved in the plastic deformation of polycrystalline ice, comparisons have often been made with the deformation of other crystalline materials (e.g. Wilson, 1986). However, because of the low crystallographic symmetry of ice and its limited glide systems (Weertman, 1983), considerable caution must be exercised in applying such comparisons.

In an attempt to analyse quantitatively the flow of natural ice masses, experimental techniques involving aggregates of ice are often used. However, to date, there are only a few experiments that successfully involve major components of shear strain (Kamb, 1972; Bouchez and Duval, 1982). In the majority of these experiments, the lattices of grains are randomly oriented before deformation and then, generally speaking, they will acquire a preferred orientation during deformation that is interpreted in terms of intracrystalline gliding and recrystallization. Although such experiments give useful insight into the geometry of deformation, and the way in which textures develop with increasing strain or temperature, detailed information on the evolution of grain microstructure and the modification of fabrics cannot be obtained from such experiments.

Another approach has been to deform thin sheets of polycrystalline ice under the optical microscope (Steinemann, 1954; Wakahama, 1964; Burg and others, 1986; Wilson and others, 1986). In this paper, three simpleshearing experiments of ice deformation at different temperatures will be described to provide an insight into the processes of intracrystalline slip and dynamic recrystallization. However, the significance of an area where recrystallization and/or grain-boundary migration is occurring cannot be comprehended unless the mechanical behaviour of an individual grain is understood. Changes in spatial distribution and grain orientation during deformation are largely governed by kinematic factors. The activated glide planes occupy characteristic preferred orientations which depend mainly on the orientation and magnitude of the bulk (mean) strain axes (Fig. 1).

To comprehend the kinematic factors controlling the ice deformation on a grain scale, the optical results will then be numerically modelled using a computer code FLAC (Fast Lagrangian Analysis of Continua; Cundall and Board, 1988); FLAC has been used to simulate fabric development in single-layer buckle folds (Zhang and others, in press b) and in polycrystalline aggregates (Zhang, 1992; Zhang and others, in press a, b). We hope that this comparative study can improve our understanding of the mechanical aspects of ice deformation as well as the associated microstructural development and 
(a)
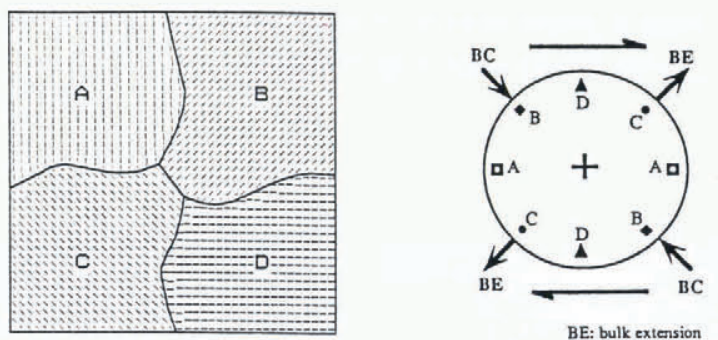

(b)

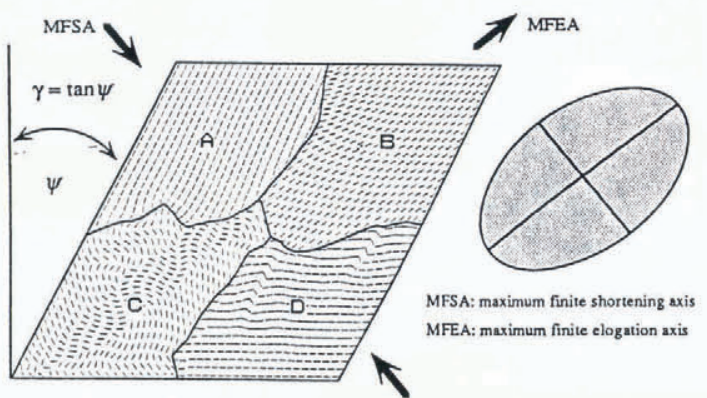

(c)

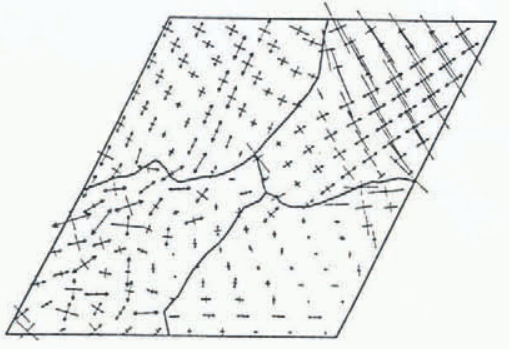

Fig. 1. Basic features of the experiments and the model. (a) Four undeformed polygonal grains ( $A, B, C$ and $D)$ with basal planes (0001) and grain boundaries oriented at a high angle to the viewing plane (left), and possible caxis orientations for each grain under the deformation frame of a dextral simple shear (right); $A$ and $D$ have an easy-glide basal-plane orientations, $B$ has a hard glide and stable orientation and $C$ has an orientation favourable for kinking. (b) Simple shear of the ice aggregate with an angular shear $\psi$ and bulk shear strain $\gamma=\tan \psi$ and showing the relationship of the maximum finite-shortening axis and shear-plane orientation; the corresponding strain ellipse is given on the right. The final basal plane of an individual grain could be anywhere between an easy-glide and hard-glide orientation. (c) Stress distribution within a grain (see text for further explanation).

also conversely provide a validity of the numerical method using FLAC.

\section{EXPERIMENTAL TECHNIQUES}

The apparatus used in this investigation has been described by Burg and others (1986) and permits the in-situ recording of the changes in a thin ice layer during progressive simple shear. A rectangular section of polycrystalline ice $25 \mathrm{~mm} \times 35 \mathrm{~mm}$ and $0.85 \mathrm{~mm}$ thick has its lower and upper surfaces confined between two fixed glass plates. The laboratory-made ice used in this study was prepared by refreezing a mixture of sieved crushed ice and distilled water as described by Wilson and Russell-Head (1982). The samples initially consisted of near equant and randomly oriented polygonal grains (mean grain-size range $2-5 \mathrm{~mm})$ with small $(0.1-0.2 \mathrm{~mm}$ ) spherical air bubbles. A summary of the experimental conditions for each experiment described in this paper has been provided by Burg and others (1986).

The simple shear undertaken was always dextral (Fig. $1 \mathrm{a}$ and $\mathrm{b}$ ) and the final shape of the bulk sample was always a parallelogram. The shear plane, shortening axis and individual grains progressively rotated with increasing angular shear so that some grains could change orientation with respect to hard-glide and easy-glide orientations (Fig. 1b). Three experiments (SS3 deformed at $-4^{\circ} \mathrm{C}$; SS6 deformed at $-5^{\circ} \mathrm{C}$ and $\mathrm{SS} 11$ deformed at $-10^{\circ} \mathrm{C}$ ) were performed over periods ranging from 3 to $5.5 \mathrm{~d}$ and produced shear strains $(\gamma)$ up to 0.75 . The thin layers of polycrystalline ice deformed homogeneously on the sample scale and there was no detectable increase in thickness of the ice layer during deformation so that the deformation was essentially plane strain. The microstructural evolution of an individual grain in an initial selected area of the sample was recorded on $16 \mathrm{~mm}$ and $35 \mathrm{~mm}$ film. As the objective lens and field of view of the microscope is fixed, grains initially visible at the start of the photography may move out of the field of view (Fig. 1) as the sample undergoes progressive simple shear and bulk rotation. Similarly, neighbouring grains not initially visible at the start of the photography may progressively move into the field of view. Prior to the commencement of the deformation, the $c$-axis orientations of the larger individual grains in any selected area were measured. Not all smaller interstitial grains could be measured because of their small size or uneven birefringence.

\section{COMPUTER-MODELLING METHODS}

FLAC is an explicit, time-marching, finite-difference computer code which is capable of mechanically modelling polycrystalline deformation involving one-slip system (see Board, 1989; Zhang and others, in press a, b, for detailed description). The model treats a polycrystalline domain as a densely packed collection of single crystals. Each crystal grain consists of an elastic-perfectly-plastic matrix containing an infinite number of slip planes (oneslip system) which also follow elastic-perfectly-plastic behaviour (the critical resolved shear-stress law). A bulk deformation of the polycrystal could dominantly be accommodated by slip-on-slip planes or by the deformation of the matrix or both, depending upon the parameter specified in the model. Slip-on-slip planes commenced when the resolved shear stress $\left(\tau_{\mathrm{r}}\right)$ on slip planes is equal to the critical resolved shear stress $\left(\tau_{c}\right)$. Similarly, the matrix deformation switches from elastic to plastic when the maximum shear stress $\left(\tau_{m}\right)$ reaches the yielding limit $\left(\sigma_{0}\right)$. Because $\sigma_{0}$ and $\tau_{\mathrm{c}}$ are totally determined by matrix cohesion $\left(C_{\mathrm{m}}\right)$ and slip-plane cohesion $\left(C_{8}\right)$, respectively, after friction angles for the matrix and slip planes are both chosen as zero setting $C_{\mathrm{m}} \gg C_{\mathrm{s}}$ leads to $\sigma_{0} \gg \tau_{\mathrm{c}}$. By so doing, we can achieve a deformation condition where slip-on-slip planes are much 
easier than the matrix deformation and therefore dominate.

Numerically, stimulating the deformation of a polycrystalline ice sample involves:
1. Mesh set-up. An area is chosen that corresponds to the selected area in the undeformed ice sample and then a finite-difference mesh to fit the geometry of ice grains in the area is generated.
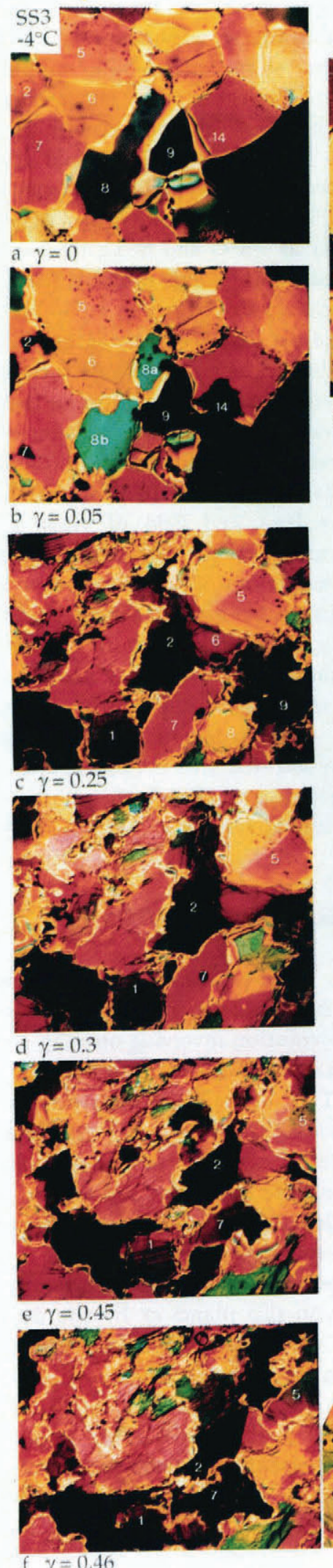

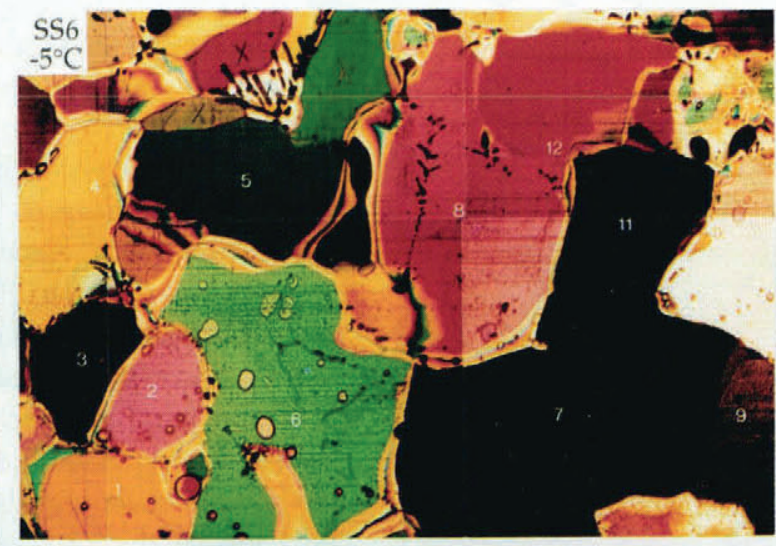

g $\gamma=0$

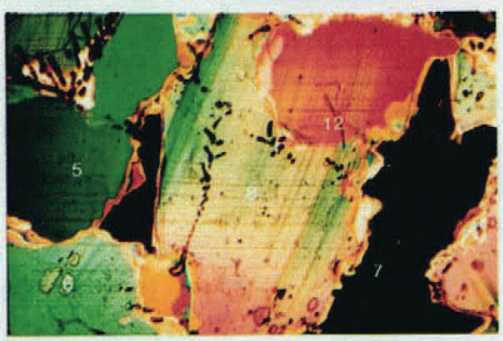

h $\gamma=0.1$

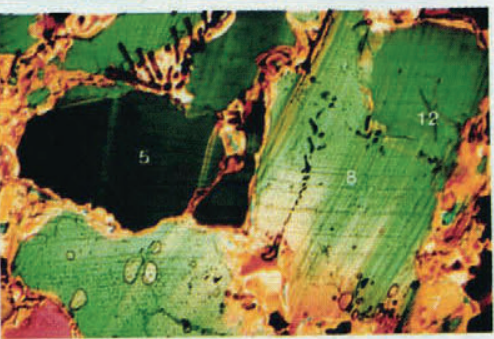

i $\gamma=0.3$
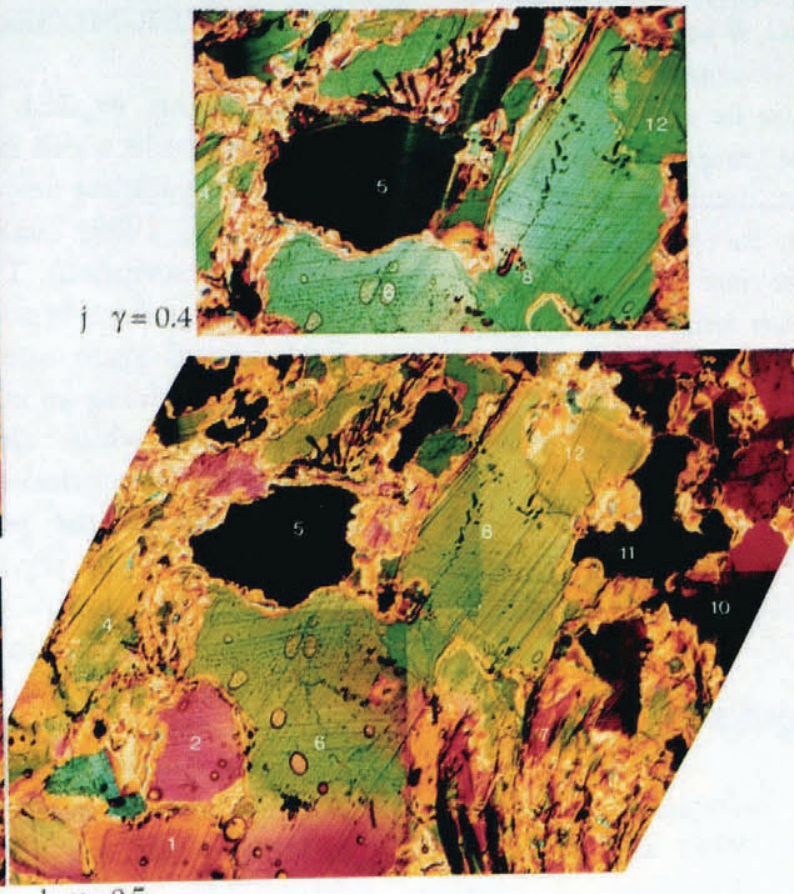

k $\gamma=0.5$

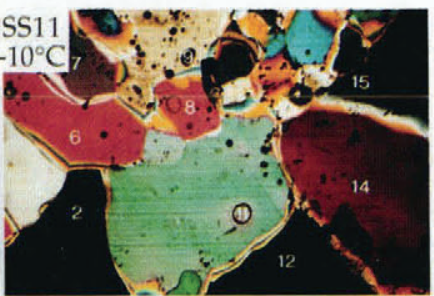

$1 \gamma=0$

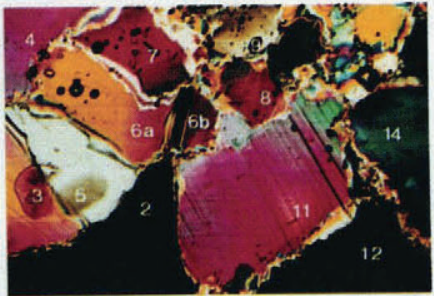

$\mathrm{m} \gamma=0.1$

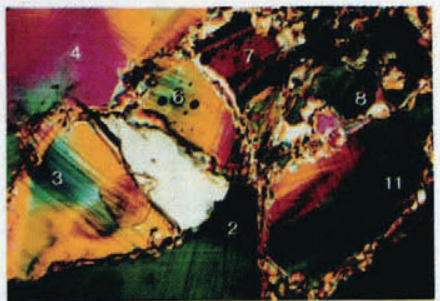

n $\gamma=0.2$

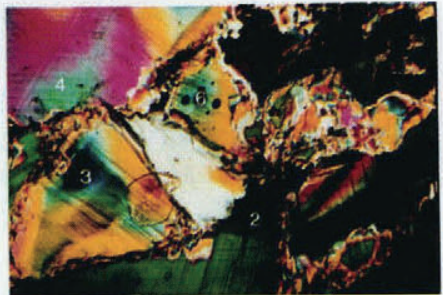

o $\gamma=0.3$

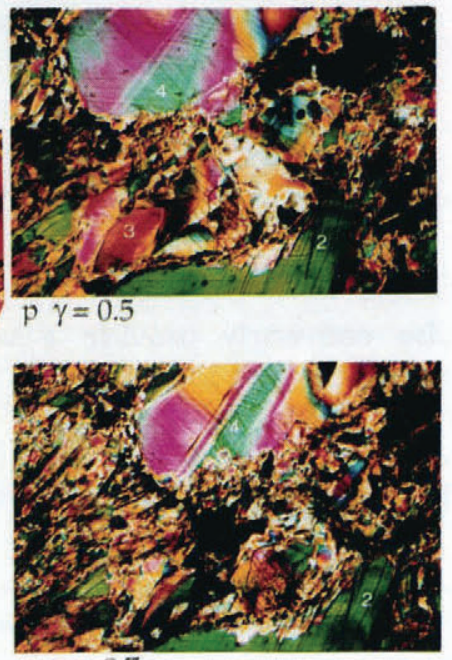

q $\gamma=0.7$

Fig. 2. Microstructural changes associated with selected areas during the dextral shear of SS3 $(a-f), \operatorname{SS} 6(g-k)$ and SS11 $(l-q)$. The average strain $(\gamma)$ is shown on the lefthand corner of the micrograph. The width of the field of view in $(a),(\mathrm{g})$ and $(\mathrm{m})$ are 8.7, 16 and $9 \mathrm{~mm}$, respectively. 
2. Lattice-orientation initialization. The slip-plane orientation was initialized for each numerical grain according to $c$-axis measurement obtained from the undeformed ice sample. Two points need to be clarified. First, c-axis orientation data were not always available for a number of ice grains in a sample owing to measurement difficulty. Therefore, we used a postulated slip-plane orientation for these grains in the numerical models; such grains are not labelled in specimens (e.g. see Fig. 3b). However, by observing slip-line orientation in the experimentally deformed aggregate (Fig. 2), it was generally possible to make a good guess for the orientation in the postulated grain. Secondly, the current two-dimensional model cannot simulate the inclination of ice basal planes directly and assumes that slip planes are always normal to the observation planes; however, we have taken slip-plane inclination into consideration indirectly by adjusting the critical resolved shear stress (next step).

3. Material property input. Material properties used in modelling are given in Table 1. Slip-plane cohesion (the critical resolved shear stress) for each grain, adjusted according to the equation

$$
\tau_{\mathrm{c}}=2 \times 10^{5} / \sin \alpha \mathrm{Pa}
$$

( $\alpha$ is the dip angle of the corresponding ice-grain basal planes), is always much smaller than matrix cohesion $\left(2 \times 10^{10} \mathrm{~Pa}\right)$ in this model. This specification ensures the domination of intragranular slip; small matrix deformation is totally elastic (see discussion in Zhang and others (in press a)).

4. Deformation. Bulk simple-shearing deformation corresponding to the experimental ice-deformation level is achieved through a series of iterative bulkstrain increments.

Stress distribution in a sample is inhomogeneous both on the inter-grain and intra-grain scales (Fig. 1c). For a particular grain, the stress level is related to the lattice orientation which determines grain-deformation features. A grain with a hard-glide orientation (e.g. B, Fig. 1c) can generally show high internal stress because of small

Table 1. Material properties used in the numerical models
Density
$917 \mathrm{~kg} \mathrm{~m}^{-3}$ (Mellor and Cole, 1982)
Young's modulus
Bulk modulus
Shear modulus

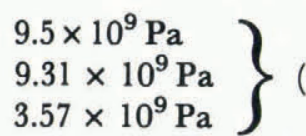
(Sanderson, 1988)
Slip-plane cohesion (CRSS) $2 \times 10^{5} / \sin \alpha \mathrm{Pa}$
Slip-plane friction
$0^{\circ}$
Matrix material cohesion
Matrix material friction

$$
2 \times 10^{10} \mathrm{~Pa}
$$

CRSS, the critical resolved shear stress; $\alpha$, the dip angle of slip planes. resolved shear stress on the slip planes and difficulty of the gliding operation. In contrast, the grains with easy-glide orientations or with the orientation favourable for the development of kink bands usually show low internal stresses. This is because in these orientations the resolved shear stress will be large enough to activate glide or internal stress will be large enough to initiate kinking while the internal stress is still quite small in comparison to other grains. Within a single grain, stress variations are observed near grain boundaries with local stress concentration at triple junctions (see triple junction between $\mathbf{A}$, B and C (Fig. 1c)).

\section{MICROSTRUGTURAL CHANGES}

\section{Experiment SS3 $\left(-4^{\circ} \mathrm{C}\right)$}

On the scale of the complete sample, there was homogeneous plastic deformation up to a shear strain of $\gamma=0.45$ and this has been illustrated by Burg and others (1986, fig. 6). However, on the scale of an individual grain the deformation is very inhomogeneous; the first obvious change is the migration of the original grain boundaries contemporaneous with the slip bands parallel to (0001) (Fig. 2a, b, c). For example, grains 6 and 9 in Figure 2a migrate into grain 8 to create two separate grains (grains $8 \mathrm{a}$ and $8 \mathrm{~b}$; Fig. 2b) that subsequently behave as individual entities during further progressive deformation (Fig. 2c and d).

Intracrystalline slip is also a dominant deformation mechanism in the early stages of deformation. However, glide within individual crystals is not uniform and depends on nearest-neighbour interactions. During gliding, the grains are undergoing crystallographic rotation, contemporaneous with the grain-boundary migration. As slip is restricted by the adjacent grains, the lattice is distorted to produce undulose extinction and kinks (e.g. grains 5 and 7 in Fig. 2c and d). At higher strains (Fig. 2e), kinks and deformation bands were particularly well developed in grains that are unsuitably oriented for easy glide. Smaller irregular grains are also nucleated adjacent to such grains (e.g. the new small grains adjacent to grains 2 and 7 in Figure 2e). Selective grain growth of these smaller irregular grains into zones of high lattice distortion, such as the kinks, reduces the area occupied by these unstable grains (Fig. 2f). The resulting microstructure then becomes dominated by grains with orientations parallel to the extension direction and the ensuing strain is dominated by further grain-boundary migration.

\section{Experiment SS6 $\left(-5^{\circ} \mathrm{C}\right)$}

This represents a coarser-grained aggregate in which the development of slip bands is prominent in all of the larger grains. Throughout the deformation, a grain such as 6 (Fig. $2 \mathrm{~g}-\mathrm{h}$ ) does not undergo a significant change in lattice orientation but accommodates the strain through continuous basal slip with only minor grain-boundary migration. Grain 6 is orientated in a hard-glide orientation with (0001) perpendicular to the constraining plates but parallel to the extension axis in the sample. 
Grains 1, 4, 8 and 12 have slip-band orientations comparable to grain 6 but dips that are oblique to the surface of the sample. These grains undergo slip and a rapid grain rotation accompanied by limited grainboundary migration. The extent of rotation can be observed by the changes in birefringence colours with the majority of these grains rotated into an orientation comparable to grain 6 .

Kinks normal to (0001) are developed in grains oriented with their basal planes parallel to the shortening direction. Grain 5 shows that, after intracrystalline glide (Fig. 2h), lattice bending occurs (Fig. 2i) that eventually develops a set of spaced kinks that are confined to this grain. On the other hand, grain 2, which has a comparable crystallographic orientation, undergoes little obvious deformation and remains as a distinct relict relatively unchanged in shape, except for minor grainboundary adjustments.

In this plane-strain deformation experiment there are grains (e.g. grain 7 in SS6; Fig. 2i) that dip oblique to the constraining glass plate. As grain 7 is constrained by rigid boundaries, it undergoes lattice bending to produce undulose extinction and an inequant sub-grain structure. In such areas, the obliquely dipping slip-band traces are not observed uniformly across the sub-grain aggregate but appear only in some of the larger misoriented subgrains. New grain nucleation and grain growth in such areas tends to occur initially at intergranular sites and not within grain interiors (intragranular sites). However, as deformation proceeds, the sub-grains appear to undergo sufficient rotation to become a distinct grain entity. From such nuclei, intragranular nucleation and grain growth occur to create a grain aggregate of strain-free grains.

The smaller interstitial grains (e.g. the three small grains between grains 5, 6 and 8) undergo initial grainboundary migration (Fig. $2 \mathrm{~h}$ ) prior to the formation of new small recrystallization grains (Fig. $2 \mathrm{i}$ and $\mathrm{j}$ ). These recrystallization aggregates, in part, tend to be in orientations that sub-parallel the extension direction. For example, the distribution of the recrystallization grains located between the large deformed grains 4, 5 and 8 (Fig. 2k) is probably controlled by the shape of their large nearest-neighbour grains.

\section{Experiment $\operatorname{SS} 11\left(-10^{\circ} \mathrm{C}\right)$}

The starting material was again a coarse-grained aggregate (Fig. 21) within which there are a number of smaller polygonal grains. There was a noticeable lack of widespread grain-boundary migration that commonly accompanied the higher-temperature deformation experiments. Minor suturing developed along all grain boundaries contemporaneous with the development of prominent slip bands (Fig. $2 \mathrm{~m}$ ). A bulging of boundaries accompanied by a minor initial grain-size increase occurred in some of the smaller grains (e.g. grain 8, Fig. 21 and $\mathrm{m}$ ) but the majority of the larger grains were reduced in grain-size. It is noticeable that, at higher strains, the majority of these smaller grains were further reduced in grain-size.

Further deformation of the larger grains was accomplished predominantly by slip with little or no boundary migration. As nearest neighbour grains began to interact, slip bands progressively bent (e.g. grain 11, Fig. $2 \mathrm{~m}$ and $\mathrm{n}$ ) and eventually formed kink boundaries (grain 6a, Fig. $2 \mathrm{~m}$ ). This process of kinking was more marked in grains that lie close to the initial shortening direction (e.g. grains 3, 6 and 11, Fig. 2m). The development of kink-band boundaries sometimes resulted in the formation of two or more apparently separate grains (e.g. grains $6 \mathrm{a}$ and $6 \mathrm{~b}$ in Fig. $2 \mathrm{~m}$ ). Grain 2 remained in a hard-glide orientation throughout the deformation (Fig. 2l-q) and developed no prominent intergranular-deformation features except for slip bands.

At higher strains, the most prominent feature of the $-10^{\circ} \mathrm{C}$ experiments is the process of new grain nucleation. Small equi-axed grains are nucleated at sites of high strain such as kink-band boundaries; for example, the kink band between grains $6 a$ and $6 b$ (Fig. $2 m$ and $n$ ) becomes a multiple-nucleation site. After nucleation, selected grains in such areas then undergo grain growth (Fig. 2o) and such grain-size increases during deformation without the development of a grain-shape preferred orientation. The new size never exceeds the smallest grain-size observed in the initial aggregate. The grain growth is not extensive and would appear to be a result of a reduction in the local strain energy. However, grain growth can cause partial or complete destruction of the pre-existing microstructure (e.g. grains 6 and 8 in Figure 2o). Intergranular sites are also prominent areas for new grain nucleation. For instance, the zone between grain 4 with grains 3, 5 and 6 (Fig. 2o and p) initially contained a few nuclei, but progressively widened as additional nuclei developed in the more deformed parts of grains 3 and 4 . After nucleation, selected grains within these areas also undergo grain growth (Fig. 2q).

New grain nucleation and a destruction of the initial grain structure is most prominent in all grains that have (0001) lying parallel to the shortening axis. If, in the initial aggregate, there are a number of neighbouring grains with comparable hard-glide orientations and grain-sizes (e.g. grains 3, 5, 6 and 7), then these are completely reduced in grain-size. Such recrystallized grains will be bounded by intensely deformed relicts of grains that experienced hard glide parallel to the shortening axis (e.g. grain 4, Fig. 2q) and of deformed and partly recrystallized grains whose $(0001)$ has always lain close to the extension axis throughout the deformation (grain 2, Fig. 2q). At shear strains of greater than $\gamma=0.5$, two distinct grain populations exist in the sample: recrystallized grains versus relict old grains. The distribution of these is governed predominantly by the distribution of larger grains with basal planes oriented for hard glide and parallel to the shear plane in the bulk sample. These contribute to defining a grain-shape fabric for which experiment SS11 has been discussed in more detail by Burg and others (1986).

\section{THE MODELS}

\section{Model of SS3}

The starting numerical model (Fig. 3a) was a direct copy of part of a larger sample illustrated in figure 6 by Burg and others (1986), in which specific grain orientations 
(a) $\quad \gamma=0$

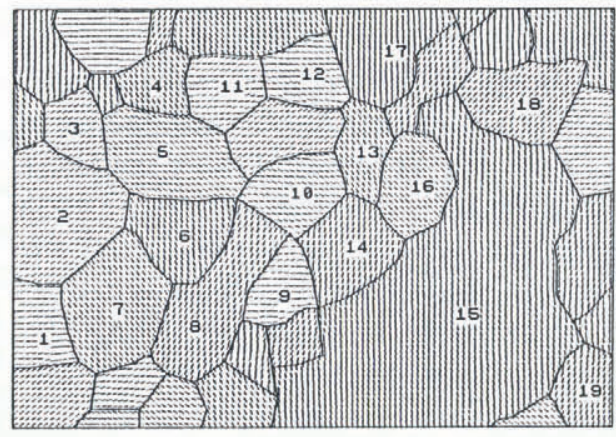

(c)

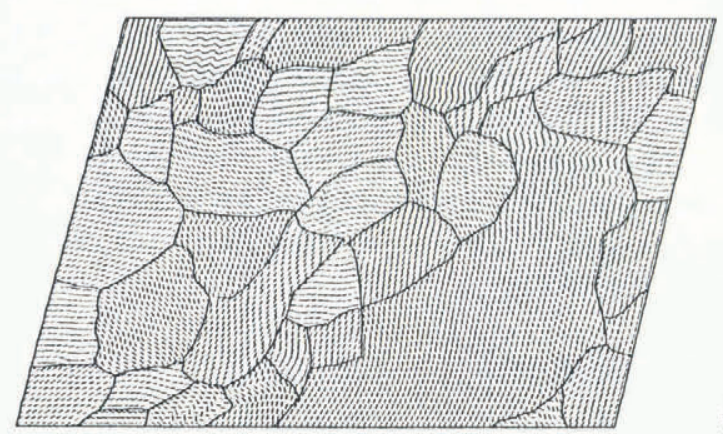

(e) $\gamma=0.45$ (b)

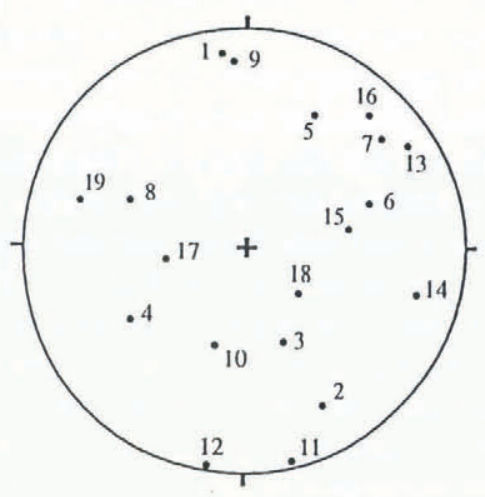

(d)

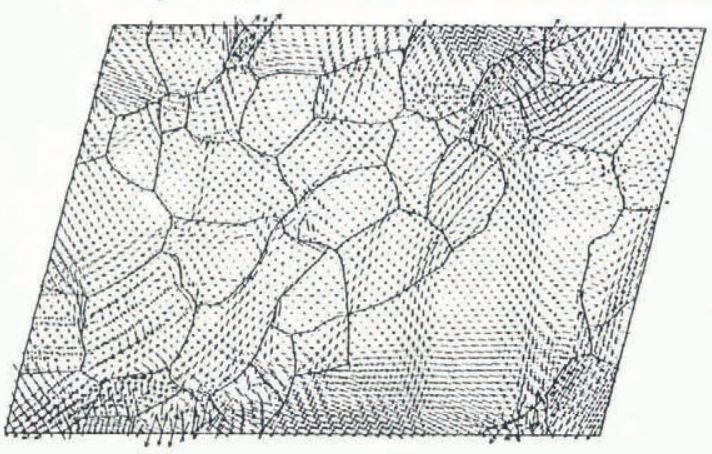

(f) $\gamma=0.25$
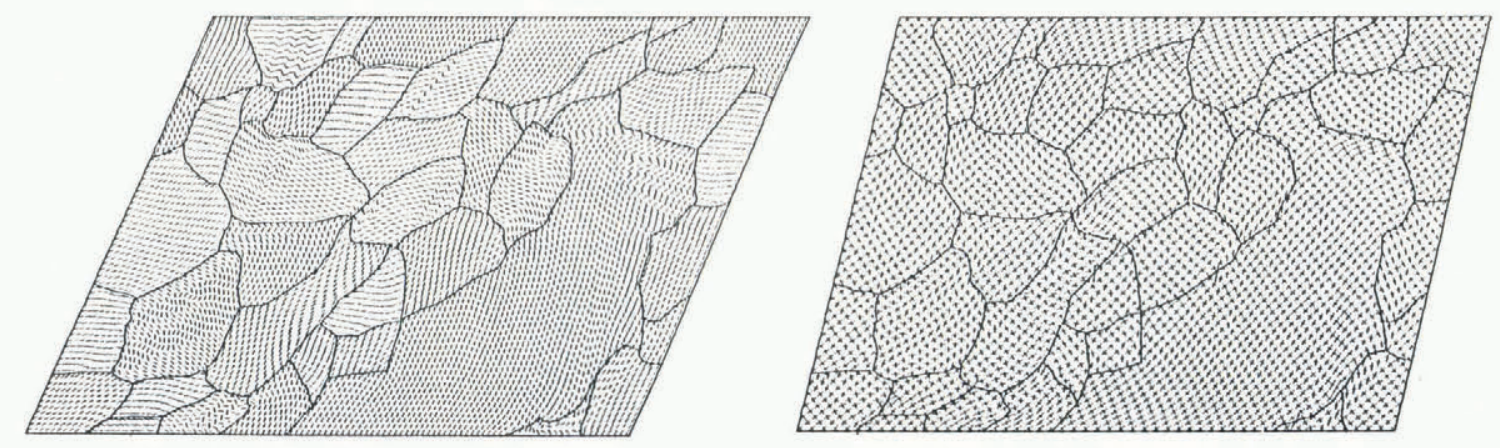

Fig. 3. Simulation of ice-deformation experiment SS3. (a) Initial slip-plane traces in the specimen generated from the ice sample shown in Figure 1a; grains are labelled from 1 through 19. (b) c-axis orientations measured for the corresponding ice grains. (c) Final slip-plane traces at a shear strain of 0.25. (d) Principal-stress distribution; the length of bars is proportional to stress magnitude and the bars with arrow heads indicate tensile stresses. (e) Final slip-plane traces at a shear strain of 0.45. $(f)$ The distribution of strain-ellipse axes.

were known (Fig. 3b). Two simulations were undertaken at shear strains $\gamma=0.25$ (Fig. 3c and f) and $\gamma=0.45$ (Fig. 3e).

Intra-grain microstructures were well developed after only a small shear strain of 0.25 (Fig. 3c), typifying the one-slip system crystallography and dextral simpleshearing deformation frame. Grains can be classified into three groups according to their initial lattice orientations and final microstructural features. The first group includes grains with initial slip-plane orientations sub-parallel to the bulk extension axis (Fig. la; e.g. 2 and 8, Fig. 3a). These grains are in a hard-glide and stable orientation with the minimum possibility of performing intragranular glide. As a result, they do not show visible intra-grain microstructures, and their small amount of lattice rotation is dominantly the result of grain interaction and the un-coaxial feature of simple shearing (Fig. 3c). The second group comprises those grains with starting slip-plane orientations sub-parallel to the bulkshortening axis (e.g. 7 and 16, Fig. 3a). These grains are also initially in hard-glide orientation because of very small resolved shear stress on the slip planes. However, this type of orientation is unstable for one-slip system under a continuous deformation. Once deformation starts, a bending moment arises along the slip planes. This bending moment, combined with the effect of grain interaction, will soon result in the nucleation of instabilities on the slip planes, which then develop into 
lattice bending/kinking and also facilitate lattice glide. Because of the mechanism described above, kink bands have been extensively developed in the grains of this group (Fig. 3c). The grains constituting the third group are those with initial slip-plane orientations sub-parallel to or sub-normal to the shear direction (e.g. 12 and 15, Fig. 3a). These grains are in easy-glide orientations with a high resolved stress on the slip planes. Therefore, they experience large intragranular slip and larger grain elongation (Fig. 3c). Local kink bands are also observed in some of these types of grains (15, Fig. 3c). This is caused by the impeding of the single slip by neighbouring grains. With the increase of bulk shear strain, the microstructural features described above are further enhanced (Fig. 3e). Particularly, kink bands become geometrically more dominant.

Stress (Fig. 3d) and strain (Fig. 3f) distributions are both inhomogeneous in the deformed specimens. The grains with a hard-glide and stable orientation exhibit relatively higher differential stress and lower strains, whereas lower differential stress and higher strains occur in the grains with an easy-glide orientation and the grains with significant development of kink bands.

\section{Model of SS6}

The numerical specimen generated here (Fig. 4a, left) reproduces a part of the coarser-grained ice sample shown in Figure $2 \mathrm{~g}$ where $c$-axis orientations for most grains have been measured (Fig. 4a, right). The simulation was undertaken to a shear strain of $\gamma=0.5$.
It is noted that microstructural results (Fig. 4b) clearly highlighted the features derived from the previous model. For example, all the grains with initial slip-plane orientations sub-parallel to the bulk extension axis (hardglide grains) are exempt from the development of intragranular microstructures (e.g. 1, 4, 6 and 8, Fig. $4 \mathrm{~b})$, whereas all the grains with slip planes initially subparallel to the bulk-shortening axis developed kink bands. Strain (Fig. 4c) and stress (Fig. 4d) results achieved here better demonstrate the dependence of strain and stress distributions upon the intragranular deformation feature in terms of lattice-glide ability. In grains with a hardglide and stable orientations, strains (Table 2) are generally very small (average strains are 1.72, 1.64 and 1.85 , respectively, for grains 1,6 and 8, Fig. 4c). In contrast, grains with easy-glide lattice orientations or with lattice orientations favourable for kinking usually show large strains (average strains are 2.94, 2.58, 2.07 and 1.98 , respectively, for grains 2, 5, 7 and 11, Fig. 4c) and small stress (Fig. 4d). Furthermore, grain boundaries, in particular triple junctions, are generally the places where strain and stress are locally strengthened or sharply changed. This is because these areas involve the strongest grain interaction arising from the need of achieving strain compatibility.

\section{Model of SS11}

Again, the numerical specimen (Fig. 5a) was created according to an ice sample with the known $c$-axis orientations, a part of which is shown in Figure 2e. A

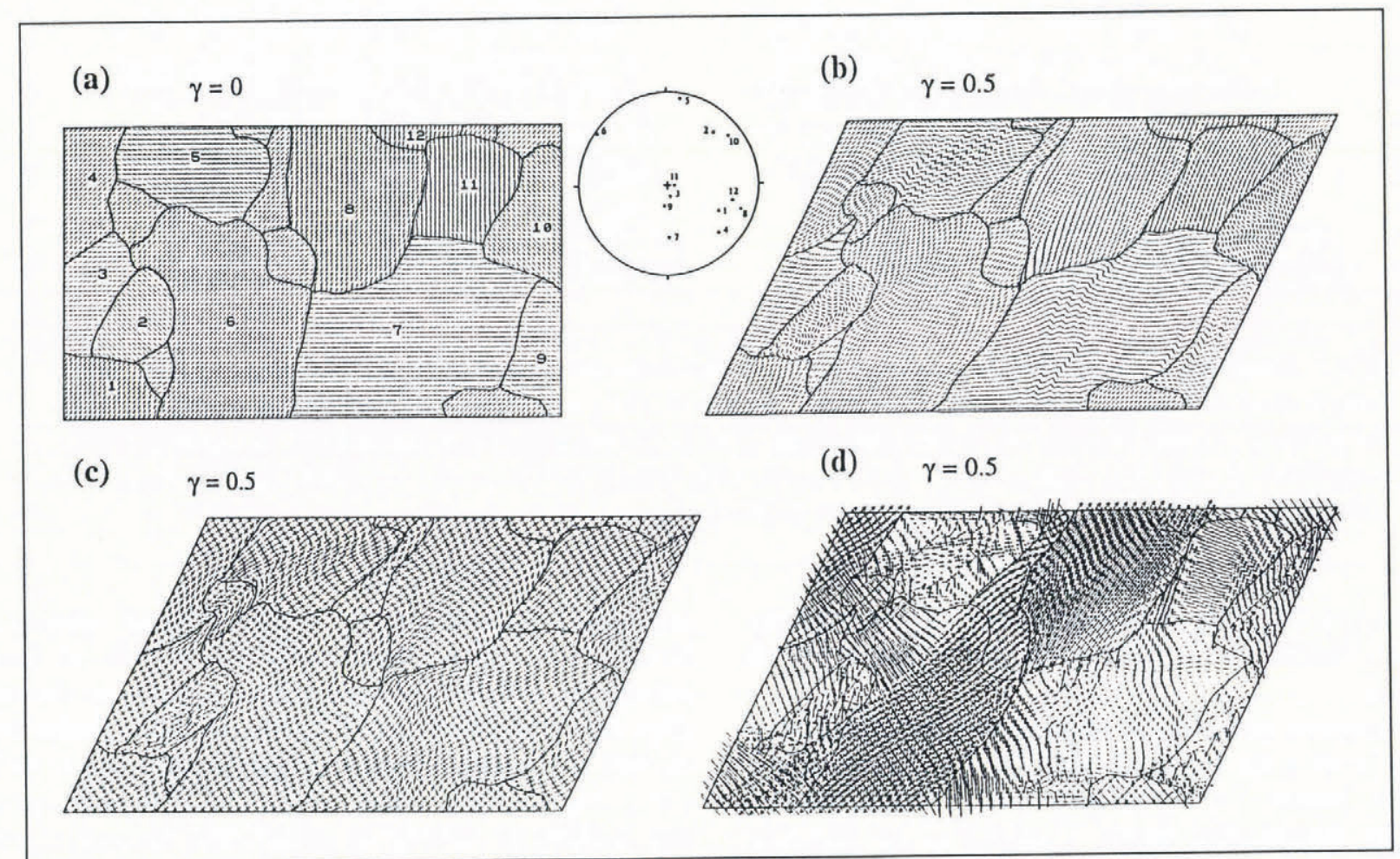

Fig. 4. Simulation of ice-deformation experiment SS6. (a) Initial slip-plane traces in the numerical polycrystalline specimen generated from the ice sample (see Fig. 1g) and c-axis orientations (right) measured for the corresponding ice grains labelled from 1 through 12. (b) Final slip-plane traces at a shear strain of 0.5. (c) The distribution of strainellipse axes. (d) The orientation and magnitude of principal stresses; the length of bars is proportional to stress magnitude and the bars with arrow heads indicate tensile stresses. 
Table 2. Grain-average strains $\left(\sqrt{\lambda_{1}} / \sqrt{\lambda_{2}}\right)$ for three numerical models

$\begin{array}{llllllllllllllllllll}\text { Grain No. } & 1 & 2 & 3 & 4 & 5 & 6 & 7 & 8 & 9 & 10 & 11 & 12 & 13 & 14 & 15 & 16 & 17 & 18 & 19\end{array}$

$\begin{array}{lllllllllllll}\text { Model 1 (SS6) } & 1.72 & 2.94 & 1.99 & 1.88 & 2.58 & 1.64 & 2.07 & 1.85 & 2.10 & 1.98 & 1.98 & 1.59\end{array}$

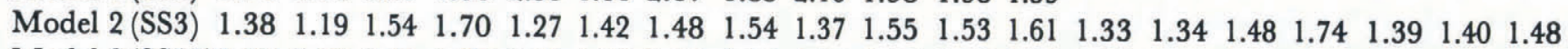

$\begin{array}{lllllllllllllllll}\text { Model } 3(\mathrm{SS} 11) 1.97 & 1.63 & 1.69 & 1.80 & 1.38 & 1.90 & 2.11 & 1.26 & 1.59 & 1.47 & 1.37 & 1.71 & 2.50 & 1.39 & 1.43 & 1.36\end{array}$

Bulk shear strain $(\gamma)$ for models 1,2 and 3 are $0.5,0.25$ and 0.3 , respectively.

simulation was performed for a shear strain of $\gamma=0.3$, and the texture and strain results are given in Figure $5 c$ and d, respectively. Clearly, these results are basically consistent with those obtained for the previous two models and simulations at higher shear strains $(\gamma=$ 0.5), highlighted the features shown in Figure 4.

\section{EXPERIMENTS VERSUS MODEL}

\section{Grains with slip plane parallel to the extension axis}

Comparison between the optical observations and the model suggest that there is excellent agreement for grains with the slip plane initially lying sub-parallel to the extension axis. These lie in a hard-glide orientation and undergo only limited glide that produces small rotations and minimal shape changes. This is partially obvious in SS6 where grains 4, 6, 8 and 12 in the model (Fig. 4b) are almost identical to the final orientation, curvature of the slip bands and shape identified in the experimentally deformed ice (Fig. 2k). The only anomaly is that grain 12 which has undergone minor rotation has migrated into grain 8. In SS11, grains such as 2 (Fig. 2q) and 10 (illustrated in figure 8 in Burg and others (1986)) are also preserved as relatively undeformed relicts in a matrix of smaller recrystallized grains. In these cases, the grains are able to deform using only intracrystalline basal glide. The juxtaposition of grains with these initial orientations does (a) $\quad \gamma=0$

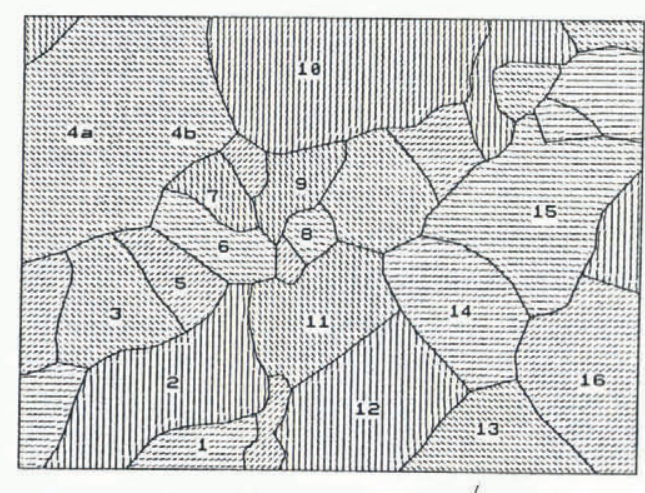

(c)

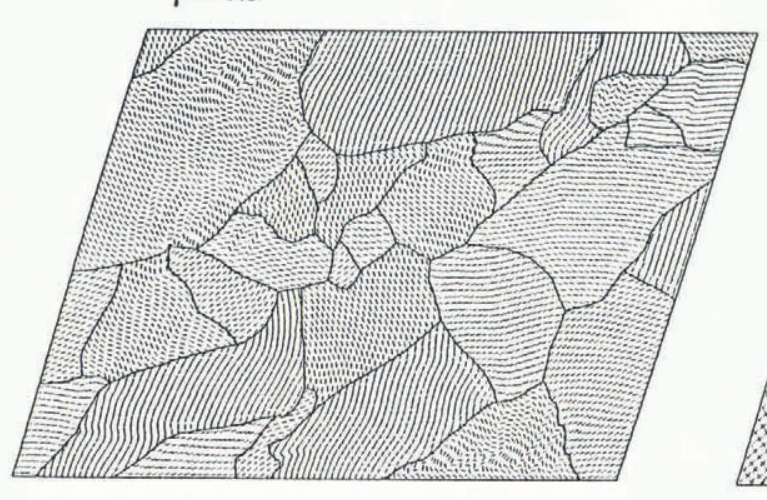

(b)

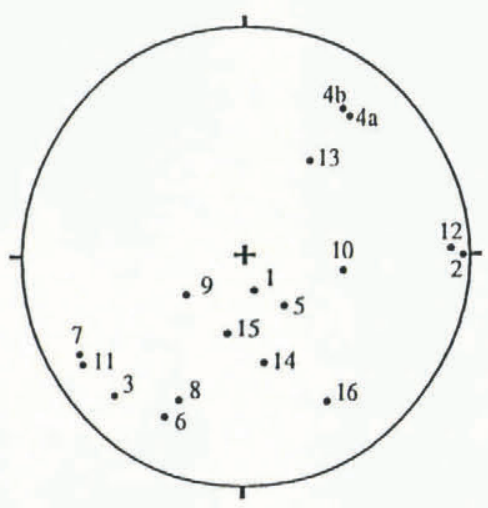

(d)

$\gamma=0.3$

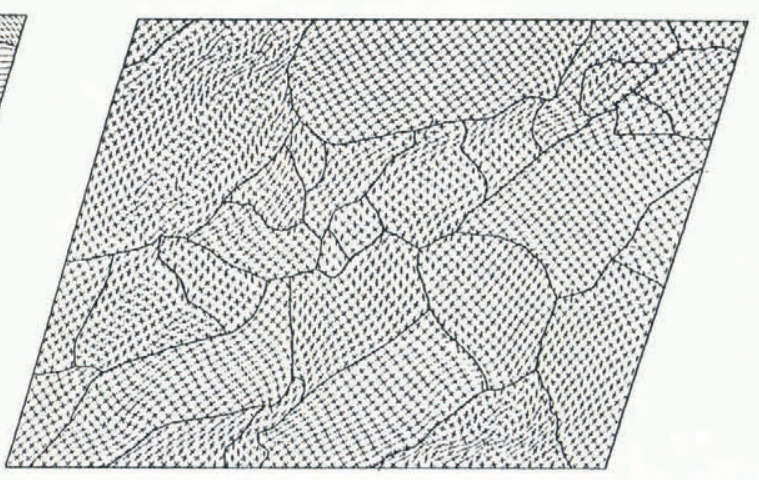

Fig. 5. Simulation of ice-deformation experiment SS11. (a) Initial slip-plane traces in the numerical specimen (see Figure $1 l$ for the correlating ice sample); grains are labelled from 1 through 16 . (b) c-axis orientations measured for the corresponding ice grains. (c) Final slip-plane traces at a shear strain of 0.3. (d) The distribution of strain-ellipse axes. 
not yield any significant changes in grain-size through processes of grain-boundary migration.

In experiment SS3, grains 2 and 8 start with slip-plane traces in hard-glide orientations, but their basal planes are oblique to the plane-strain section. The model predicts (Fig. 3) that these two grains should only undergo glide and maintain their individuality. However, during an experiment (Fig. 2b-f), these grains undergo rotation accompanied by extensive grainboundary migration. There is dissection of grain 8 into two individuals ( $8 \mathrm{a}$ and $8 \mathrm{~b}$, Fig. $2 \mathrm{~b}$ ) with $8 \mathrm{a}$ eventually disappearing, being consumed by neighbouring grains through a process of grain-boundary migration. This effect may arise because the grains are metastable and need to rotate. The only way to accommodate this rotation between neighbouring grains is to involve grainboundary migration. By this process, part of the grain is moved from the metastable orientation to allow rotation of the relict part to a stable single-slip end orientation.

\section{Grains with slip plane parallel to the shortening axis}

These grains undergo the most dramatic set of intragranular changes with all models predicting that the majority of these grains will deform by glide followed by sharp lattice bending to produce kink bands. The simplest correspondence between model and the higher-temperature experiments is displayed in SS6 (Fig. $2 \mathrm{~g}-\mathrm{k}$ ) where grain 5 is unrecrystallized but bent into a series of open kinks. The model predicts such a microstructure, with a negligible stress concentration (Fig. 4d) in the grain and a zone of high strain perpendicular to the basal plane (Fig. $4 c)$. If the basal plane is inclined rather than perpendicular to the section (e.g. grain 7 SS66, Fig. $2 \mathrm{~g}-\mathrm{k}$ ), then the model predicts the formation of irregular kinks (Fig. 4b) and the corresponding strain (Fig. 4c) and stress (Fig. 4d) distributions are also markedly non-uniform across the grain. This is confirmed in part by the experiments where there is prominent undulose extinction (Fig. 2k). However, the grain is obviously metastable and unfavourably oriented for the development of kink bands; instead intragranular nuclei are produced and further strain is accompanied by grain growth.

In the lower-temperature experiment SS11, there is a strong correspondence between both the model (Fig. 5) and experiment (Fig. 21-0). For comparable strains, grains such as 3, 4, 6, 7 and 11, where (0001) slip bands lie parallel to the shortening axis, all undergo kinking as predicted in the model. Thus, a simple geometrical correlation can be made up to shear strains of $\gamma=0.3$ as there is negligible recrystallization and the preservation of the intergranular regions. This is, therefore, unlike all the other models as there are no other competing microstructural processes, such as grain-boundary migration. At higher strains, the model predictions include further amplification of the kinks, but no other inter- or intragranular change, whereas in the experiments, as the grains continue to kink, extensive nuclei are developed at sites of high strain (Fig. 2q) and many pre-existing grains are replaced by smaller recrystallized grains.

\section{Grains oriented for easy glide}

In the models, all grains that initially lay in an easy-glide orientation, for example, grains 6, 9, 10 and 17 in SS3 (Fig. 3), are progressively elongated parallel to the bulk extension axis (Fig. 1). The models predict that with increasing strain there will be gentle bending of the (0001) glide planes. However, in the comparable experiments, there is little lattice bending, instead, these are the first grains to undergo extensive grain-boundary migration and recrystallization (Fig. 2a-c).

\section{Nearest-neighbour grain interactions}

The most important consequence of grain interactions in the experiments is the nucleation and growth of small recrystallized grains. This depends on a combination of factors that include size, shape anisotropy and the mismatch of basal planes across grain boundaries. This is particularly well developed where grains lying in a hardglide orientation constitute hard spots in the ice as their basal planes are parallel to the shortening direction. For example, grains 2 and 5 in SS6 (Fig. 2k) have undergone minimal deformation and remained as distinct relicts, but their boundaries parallel to the bulk extension direction are intensely recrystallized. In the simulated models, it is predicted that (0001) in grain 2 will kink; however, in the experiments, the slip bands are undeformed. Thus, in experiment SS6 grain 2 must experience less stress and strain than predicted in the model. Similarly, the model predicts that the nearest-neighbour grains would have high localized stresses and strains in the intergranular region. The recrystallization process is therefore a consequence of the high internal strain-energy difference between the rigid grain and its nearest neighbour.

As the mis-match across a grain boundary is reduced, there is a consequent drop in the magnitude in the stress in the model. This stress can be reduced if the grain rotates at the same time that it deforms in basal glide. The adjustment between the two nearest-neighbour grains is taken up by grain-boundary migration. An example of this was seen with grain 12 in experiment SS6 (Fig. $2 \mathrm{~g}-\mathrm{k}$ ).

Triple-point junctions between neighbouring grains in all models tended to be the area of highest localized stress and strain differences. In the higher-temperature experiments, these are always the sites where grain-boundary migration is first initiated. The triple-point junction in the grain with an easy-glide orientation is often migrating at the expense of grains in hard-glide orientations (grain 8, Fig. 2b). The extent of boundary adjustments observed in the experiments is certainly not predicted in the models. In the lower-temperature experiments (SS11) the triplepoint junctions are also the sites for the first and also most extensive recrystallization. This is particularly marked where there are small interstitial grains.

\section{CONGLUSIONS}

Deformation in the polycrystalline ice occurs predominantly by glide on a single-slip plane (0001) within the constraints of the plane-strain boundary conditions imposed on the sample. This slip can accommodate the 
deformation by a transformation in grain shape. With increasing strain, all grains become elongated parallel to the maximum finite-elongation axis. However, a degree of non-uniformity of deformation can occur within a grain, particularly if the crystallographic orientation is oblique to the maximum finite-elongation axis. We shall now assess what impact this may have on the microstructural behaviour of the ice aggregate, as seen by comparing the experiments with the models.

Kinks, undulose extinction and sub-grain structures have been extensively developed in all the experimentally deformed specimens. The formation of these structures is closely related to initial slip-plane orientations. Kinks only develop in the grains with slip planes initially parallel, or at a low angle to the shortening direction. In these orientations, large bending moments are present and this is the only way an individual grain can accommodate the shortening. Undulose extinction or gentle lattice bending is particularly well developed where metastable grains accommodate strain variations between neighbouring grains through a process of slipband flexing as the aggregate undergoes bulk rotation during the simple-shearing process. Intergranular subgrain development is a second-order effect which originates as a geometrical consequence of having shallow-dipping slip planes constrained by the rigid two-dimensional boundaries of the sample. The bending moment of the grain is restricted and there are no interactions with neighbouring grains. Instead, small intergranular lattice misorientations produce sub-grains that may undergo boundary migration to produce a recrystallized grain aggregate.

At lower temperatures $\left(-10^{\circ} \mathrm{C}\right)$ and small shear strains $(\gamma \leq 0.3)$, precisely the same microstructures are obtained from both the experiments and the computer models. As the shear strain increases, new grain nucleation and grain growth destroy the pre-existing microstructure. This nucleation preferentially occurs at intergranular sites and within highly strained grains, e.g. along kink bands. The model predicts this situation, the recrystallization and grain-boundary migration occurring where there is evident stress-strain localization and variation between neighbouring grains.

A departure from the model is that, at higher temperatures, the intracrystalline slip is accompanied by extensive grain-boundary migration. This is particularly obvious in grains oriented in an easy-glide orientation. In these cases, grain-boundary migration probably plays an important role in the elimination of the grains unsuitably oriented to deform in a hard-glide orientation. Some small new grains that undergo extensive grain growth are also observed near triple junctions, while the corresponding model displays evident stress-strain variations at these sites.

\section{ACKNOWLEDGEMENTS}

This research was supported by grants from the Australian Research Grants Committee. J. C. Mitchell is thanked for technical assistance in undertaking the experiments.

\section{REFERENCES}

Alley, R.B. 1992. Flow-law hypotheses for ice-sheet modeling. $f$. Glaciol., 38(129), 245-256.

Board, M. 1989. FLAC: fast Langrangian analysis of continua. User manual, version 2.20. Software summary. Minneapolis, MN, Itasca Consulting Group, Inc.

Bouchez, J.L. and P. Duval. 1982. The fabric of polycrystalline ice deformed in simple shear: experiments in torsion, natural deformation and geometrical interpretation. Textures Microstruct., 5, 171190.

Burg, J.P., C.J.L. Wilson and J.C. Mitchell. 1986. Dynamic recrystallization and fabric development during the simple shear deformation of ice. 7. Struct. Geol., 8(8), 857-870.

Cundall, P.A. and M. Board. 1988. A microcomputer program for modelling large-strain plasticity problem. In Swobada, C., ed. Numerical methods in geomechanics. Proceedings of the 6th International Conference on Numerical Methods in Geomechanics. Rotterdam, Balkema, 2101-2108.

Fukuda, A., T. Hondoh and A. Higashi. 1987. Dislocation mechanisms of plastic deformation of ice. 7. Phys. (Paris), 48, Collog. C1, 163-173. (Supplément au 3.)

Hooke, R. LeB. and P.J Hudleston. 1978. Origin of foliation in glaciers. f. Glaciol., 20(83), 285-299.

Kamb, B. 1972. Experimental recrystallization of ice under stress. In Heard, H. C., I. Y. Borg, N. L. Carter and C. B. Raleigh, eds. Flow and fracture of rocks. Washington, DC, American Geophysical Union, 211-241. (Geophysical Monograph 16.)

Lliboutry, L.A. 1987. Very slow flow of solids: basics of modelling in geodynamics and glaciology. Dordrecht, Martinus Nijhoff Publishers.

Mellor, M. and D. M. Cole. 1982. Deformation and failure of ice under constant stress or constant strain-rate. Cold Reg. Sci. Technol., 5(3), 201-219.

Sanderson, T.J. O. 1988. Ice mechanics; risks to offshore structures. London, Graham and Trotman

Steinemann, S. 1954. Results of preliminary experiments on the plasticity of ice crystals. F. Glaciol., 2(16), $404-413$.

Wakahama, G. 1964. On the plastic deformation of ice. V. Plastic deformation of polycrystalline ice. Low Temp. Sci., Ser. A 22, 1-24. [In Japanese with English summary.]

Weertman, J. 1983. Creep deformation of ice. Annu. Rev. Earth Planet Sci., 11, 215-240.

Wilson, C.J. L. 1986. Deformation induced recrystallization of ice: the application of in situ experiments. In Hobbs, B. E. and H. C. Heard, eds. Mineral and rock deformation laboratory studies. Washington, DC, American Geophysical Union, 213-232. (Geophysical Monograph 36.)

Wilson, C.J.L. and D.S. Russell-Head. 1982. Steady-state preferred orientation of ice deformed in plane strain at $-1^{\circ} \mathrm{C}$. $\mathcal{F}$. Glaciol., 28(98), 145-160.

Wilson, C. J. L., J. P. Burg and J. C. Mitchell. 1986. The origin of kinks in polycrystalline ice. Tectonophysics, 127(1-2), 27-48.

Zhang, Y. 1992. Fabric development and deformation behaviour in polycrystalline aggregates containing one slip system. (Ph.D. thesis, Monash University, Australia.)

Zhang, Y., B. E. Hobbs and M.W. Jessell. 1993. Crystallographic preferred orientation development in a buckled single layer: a computer simulation. J. Struct. Geol., 15, 265-276.

Zhang, Y., B. E. Hobbs and A. Ord. In press a. A numerical simulation of fabric development in polycrystalline aggregates with one slip system. F. Struct. Geol.

Zhang, Y., B. E. Hobbs and M. W. Jessell. In press b. The effect of grain boundary migration sliding on fabric development in polycrystalline aggregates. F. Struct. Geol.

The accuracy of references in the text and in this list is the responsibility of the authors, to whom queries should be addressed. 